\title{
Relação do Bem-Estar Subjetivo, Estratégias de Enfrentamento e Apoio Social em Idosos
}

\author{
Relationships of Subjective Well-Being, Coping Strategies \\ and Perceived Social Support in the Elderly
}

\author{
Miriam Teresa Domínguez Guedea ${ }^{a^{*}}$, Francisco José Batista de Albuquerque, \\ Bartholomeu Tôrres Tróccoli ${ }^{c}$, José Angel Vera Noriega ${ }^{d}$, \\ Magno Alexon Bezerra Seabra ${ }^{e} \&$ Rosario Leticia Domínguez Guedea ${ }^{a}$ \\ ${ }^{a}$ Universidad de Sonora, Hermosillo, México, ${ }^{b}$ Universidade Federal da Paraíba, João Pessoa, Brasil, \\ 'Universidade de Brasília, Brasília, Brasil, ${ }^{d}$ Departamento de Desarrollo Humano y Bienestar Social, Hermosillo, México, \\ ${ }^{e}$ Centro Universitário de João Pessoa, João Pessoa, Brasil
}

\begin{abstract}
Resumo
Foram analisadas as relações das estratégias de enfrentamento, apoio social e variáveis sócio-demográficas com o bem-estar subjetivo de uma amostra de idosos. O bem-estar foi definido pela satisfação com a vida, os afetos positivos e afetos negativos. Participaram 123 idosos (média $=67,1$; desvio padrão = 6,1), residentes em João Pessoa, Brasil. Análises de regressão hierárquica revelaram que (a) a satisfação com a vida é maior em mulheres, nas pessoas que recebem pensão, as pessoas que estão satisfeitas com o apoio recebido, as pessoas que dão apoio aos outros e nas pessoas que enfrentam os problemas de forma direta e uma re-avaliação positiva; (b) os afetos positivos aumentam com a satisfação do apoio recebido e com o enfrentamento direto e re-avaliativo e a diminuição do enfrentamento de esquiva e (c) os afetos negativos diminuem ao dar apoio e aumentam com o enfrentamento de esquiva. Os resultados mostram as diferenças dos componentes cognitivo e afetivo do bem-estar e apontam a necessidade de investigar a autonomia funcional do idoso.

Palavras-chave: Bem-estar subjetivo; enfrentamento; apoio social; idosos.
\end{abstract}

\begin{abstract}
Subjective well-being was studied in a sample of the elderly by analyzing the relationship among coping strategies, social support, and social-demographic variables. Well-being was defined by life satisfaction and positive and negative affect. There were 123 elderly participants (mean=67.1; standard deviation=6.1) who were residents in João Pessoa, Brazil. Hierarchical regression analyses revealed that (a) life satisfaction is higher in: women, people who receive pensions, people who are satisfied with the support they receive, people who give support to others, and people who cope with problems directly and by re-appraising the situation in a positive way; (b) positive affect increases with the satisfaction of the support received, with direct and reappraisal coping, and with the decrease of coping by avoidance, and (c) negative affect decreases with giving support and increases with coping by avoidance. The results show the differences of the cognitive and affective components of well-being and identify the need to investigate the functional autonomy of the elderly. Keywords: Subjective well-being; coping; social support; elderly.
\end{abstract}

Nas últimas décadas, tem-se registrado um crescimento mundial nos índices de envelhecimento demográfico, em conseqüência da redução das taxas de natalidade e do aumento na expectativa de vida (García-Sanz \& Saco, 1995; Instituto Brasileiro de Geografia e Estatística [IBGE], 2002). O Brasil iniciou o novo século com uma população idosa crescendo duas vezes mais do que a sua população total e, segundo as estimativas para os próximos 20 anos,

\footnotetext{
"Endereço para correspondência: SCLN, 209, Bl. A, apto. 218, Brasília, DF, 70554-510. E-mail miriamd@sociales.uson.mx

Este trabalho foi realizado com o apoio da Universidade de Sonora, do Núcleo de Pesquisa em Desenvolvimento Rural e Vulnerabilidade Social da Universidade Federal da Paraíba e do Laboratório de Pesquisa em Avaliação e Medida do Instituto de Psicologia da Universidade de Brasília.
}

quase $13 \%$ da população brasileira será constituída por idosos (IBGE; Ministério da Saúde, 2001). Segundo as projeções da Organização Mundial da Saúde, no ano 2025 o Brasil será o sexto país com o maior número de pessoas com 60 anos ou mais.

O aumento na expectativa de vida provoca mudanças na estrutura da população, criando oportunidades não apenas para que as pessoas vivam mais anos, mas também para melhorar a qualidade de vida desses anos. Dentro da perspectiva psico-social, considera-se a qualidade de vida como o conjunto de avaliações que as pessoas fazem das áreas significativas das suas vidas (Martínez \& García, 1994). Estas avaliações podem ter determinantes objetivos (ex., questões sociais, demográficas, políticas, econômicas) e subjetivos (Ranzjin \& Luszcz, 2000). A percepção que as pessoas têm do seu bem-estar é um dos aspectos subjetivos 
vinculados à qualidade de vida (Martínez \& García, 1994; Ormel, Lindenberg, Steverink \& Vonkorf, 1997).

O bem-estar subjetivo (BES) está associado a um envelhecimento mais saudável (Gracia, 1997), é um indicador de saúde mental e também sinônimo de felicidade, ajuste e integração social (Anguas, 1997). O BES também se associa negativamente com sintomas depressivos e doenças físicas (Martínez \& García). O bem-estar subjetivo é, portanto, um indicador importante do nível de adaptação na terceira idade, estágio do desenvolvimento que já corresponde, na estrutura demográfica atual, a aproximadamente vinte e cinco por cento da vida das pessoas. O bem-estar subjetivo é composto pelos componentes afetivo e cognitivo (Albuquerque \& Tróccoli, 2004; Bohlander, 1999). O componente afetivo é identificado como um equilíbrio entre os afetos positivo e negativo (Emmons, 1986; Krause, Ingersoll-Dayton, Ellison \& Wulf, 1999; Ranzjin \& Luszcz, 2000). Os afetos positivos são emoções prazerosas, tais como fruição e a felicidade, enquanto que os afetos negativos são sentimentos desagradáveis ou emoções tais como a tristeza e o medo (Emmons). Já o componente cognitivo é considerado como uma avaliação entre as metas desejadas e as alcançadas (Ranzjin \& Luszcz), e tem sido identificado como satisfação com a vida (Chatters, 1988; Emmons), compreendida como um processo de julgamento e avaliação geral da própria vida.

As teorias físico-biológicas, psicológicas e sociológicas do envelhecimento indicam que o idoso precisa adaptar-se a situações sociais e condições físicas típicas da terceira idade (Agulló, 1999). O processo de envelhecimento caracteriza-se por uma perda gradual das capacidades funcionais comprometendo a autonomia da pessoa para satisfazer suas necessidades cotidianas. As perdas e conseqüências negativas, no entanto, não implicam em níveis de dificuldades adaptativas iguais entre todos os idosos. Pesquisas indicam diversos e variados tipos de adaptação das pessoas à velhice. Chatters (1988), por exemplo, encontrou em uma amostra de idosos nor te-americanos, correlação negativa entre a idade e a percepção dos problemas da vida, ou seja, na medida em que a idade aumenta, a percepção dos problemas da vida diminui. Este resultado é semelhante ao encontrado por Martínez e García (1994) em idosos espanhóis. Para estes autores o passar dos anos não deteriora a auto-avaliação da saúde porque os idosos avaliam a saúde como boa quando ela é sufi-ciente para viver. A saúde não é percebida como ausência total de enfermidades, fruto da comparação da situação presente com um padrão ideal, mas sim uma avaliação ligada a expectativas sobre o que esperar da velhice. No entanto, para muitas pessoas a velhice é vivida como uma etapa cheia de acontecimentos adversos que afetam o estado de ânimo e a percepção de bem-estar em geral e é marcada pela vulnerabilidade e fragilidade do organismo e pela dependência econômica, social, afetiva e física (Gracia, 1997).

Independentemente do tipo adaptativo predominante em um determinado grupo de idosos, a pessoa que envelhece transita num contínuo que vai da adaptação a não adaptação às condições próprias desta etapa da vida. Quais são os recursos pessoais que permitem que idosos consigam se adaptar em diferentes graus a um processo de envelheci- mento potencialmente gerador de estresse? Surpreendentemente, fatores demográficos (sexo, raça, educação, renda e estado civil), exercem quase nenhuma influência sobre o bem-estar subjetivo. De fato, tomando-as todas em conjunto, raramente explicam mais de $10 \%$ da variância no equilíbrio dos afetos ou na satisfação com a vida. As mais altas diferenças são apenas observadas em relação à renda e ao nível de escolaridade (Andrews \& Robinson, 1991; Bohlander, 1999).

Dentre as variáveis psicosociais, as estratégias de enfrentamento apresentam uma contribuição significativa para o bem-estar subjetivo dos idosos. São mecanismos que os indivíduos utilizam para minimizar os efeitos do estresse, resolvendo ou manejando o problema com o objetivo de voltar à normalidade de funcionamento pessoal o mais rápido possível (Aldwin, Sutton \& Lachman, 1996). As estratégias de enfrentamento podem ter efeitos amortecedores de condições adversas experimentadas pelo idoso. Por exemplo, há quem retire benefícios na exposição a eventos estressantes ao vivenciar um aumento do sentimento de controle, aumento da auto-estima, maior capacidade empática, aumento da proximidade com outras pessoas, mudanças nos valores e maior auto-entendimento e sabedoria (Aldwin et al.).

Os recursos de enfrentamento tanto são características individuais quanto estão associados ao apoio social percebido pelo indivíduo (Abril, 1998). O apoio ou suporte social - manifesto através do uso de equipamentos comunitários, redes sociais e relações íntimas, por exemplo -, também permite satisfazer necessidades (instrumentais e expressivas) em situações cotidianas e de crise (Gracia, 1997). Os recursos pessoais do idoso são muito importantes para sua adaptação no processo em que acontecem as perdas. Tais recursos podem ser promovidos, apoiados ou inclusive colocados nos espaços de relações sociais significativas, mediante o acesso a vínculos sociais compensatórios que possam proteger as pessoas com idade avançada dos sentimentos negativos derivados das perdas.

Assim, o apoio social relaciona-se com o bem-estar psicológico e a saúde mental (Jung, 1997; Krause \& BorawskiClark, 1995), está negativamente correla-cionado com depressão e estresse (Jung, 1997; Newson \& Schultz, 1996) e com a sintomatologia física, associando-se com a redução de riscos de mortalidade (Felton \& Shinn, 1992) e sendo preditor significativo dos recursos pessoais de enfrentamento dos problemas (Newson \& Schultz).

Considerando, portanto, as possíveis relações entre as variáveis examinadas, são dois os objetivos principais deste estudo: (a) descrever e analisar as três dimensões do bem-estar subjetivo e (b) investigar o valor preditivo das estratégias de enfrentamento e do suporte social sobre o bem-estar subjetivo de uma amostra de idosos. Especificamente, espera-se um valor preditivo significativo das variáveis estratégias de enfrentamento dos problemas e apoio social percebido sobre cada uma das três dimensões, bem como diferenças significativas entre estes três componentes do bem-estar subjetivo. 


\section{Método}

\section{Participantes}

Participaram deste estudo 123 (cento e vinte e três) pessoas com idades entre 60 e 93 anos (média $=67,1$; desvio padrão $=6,1)$ residentes na cidade de João Pessoa, Paraíba, que preencheram os seguintes requisitos: (a) ser alfabetizado funcional; (b) ter uma renda familiar entre $\mathrm{R} \$ 800,00$ e $\mathrm{R} \$ 1.500,00$ (oitocentos a mil e quinhentos reais, equivalentes a aproximadamente três a quatro salários mínimos) e; (c) ter morado na cidade do estudo pelo menos durante 20 anos. Devido às dificuldades em se obter uma amostra de tamanho adequado, foram incluídas todas as pessoas com 60 anos ou mais que se dispuseram a participar da pesquisa, embora esta faixa etária possa ser considerada demasiadamente ampla. Quanto à escolaridade, $25,2 \%$ da amostra estudou o primeiro grau completo, $20,3 \%$ terminou o segundo grau e $25,2 \%$ completou o terceiro grau. Dos homens, $4,8 \%$ recebe pensão, e $74,2 \%$ recebe o benefício da aposentadoria. No caso das mulheres, 23 ,\% recebem pensão e $46 \%$ são aposentadas. Para fins de análise, a amostra foi dividida em quatro grupos resultantes do cruzamento das variáveis sexo e trabalho.

\section{Instrumentos}

Foi utilizada a Escala de Bem-estar Subjetivo, validada na população geral residente em João Pessoa, Paraíba. A escala se divide em duas partes: afetos e satisfação com a vida. Os itens relativos aos afetos são acompanhados por escalas tipo Likert de cinco pontos que medem a freqüência $(1=$ nunca $-5=$ sempre $)$ e a intensidade ( $1=$ nada $-5=$ muito $)$ na vivência de um conjunto de experiências afetivas. Esses itens representam dois fatores que explicam $34 \%$ da variância: (a) afetos negativos (nove itens; á = 0,91); (b) afetos positivos (oito itens; á = 0,88). Já os itens que mensuram a satisfação com a vida também estão acompanhados de uma escala Likert ( $1=$ nada satisfeito $-5=$ muito satisfeito) $\mathrm{e}$ representam cinco fatores que explicam $48,6 \%$ da variância: (a) satisfação com os amigos (seis itens; á = 0,84); (b) satisfação com a família (cinco itens; á = 0,84); (c) satisfação com a autonomia pessoal (quatro itens; á $=0,81$ ); (d) satisfação com recursos econômicos (quatro itens; á = 0,79) e; (e) satisfação com a religião (três itens; á $=0,86$ ).

Para avaliar o enfrentamento, foi aplicada a Escala de Estratégias de Enfrentamento também validada na população de João Pessoa. A escala é composta de 39 itens acompanhados de escalas tipo Likert de cinco pontos $(1=$ nunca $-5=$ sempre), que medem a frequiência com a qual a pessoa lida com problemas com os amigos, familiares, parceiros (a), com a saúde e com a vida em geral. Os dois fatores subjacentes aos itens dessa escala, que explicam $54 \%$ da variância total, são: (a) enfrentamento direto e reavaliativo (19 itens; á $=0,98)$ e (b) enfrentamento de esquiva (20 itens; á $=0,89$ ) O fator enfrentamento direto e re-avaliativo diz respeito aos esforços cognitivos e comportamentais da pessoa para lidar com seus problemas, bem como às mudanças cognitivas realizadas para mudar sua percepção do problema em um sentido mais positivo. Já o segundo fator, enfrentamento de esquiva, diz respeito às tentativas, cognitivas ou comportamentais realizadas na tentativa de se evitar ou escapar do problema.

Para coletar os dados referentes ao apoio social, elaborou-se uma entrevista estruturada com base nos fundamentos conceituais do construto. Das entrevistas foram retiradas as seguintes informações: (a) freqüência de contatos sociais (soma do número de pessoas com as quais o idoso convive diária, semanal ou mensalmente); (b) percepção de apoio (soma das respostas afirmativas que o participante deu sobre ter quem lhe apóie em problemas emocionais, práticos e informacionais); (c) satisfação com o apoio (média das respostas, expressas em porcentagens, dadas a várias questões sobre a satisfação com o apoio recebido) e, (d) provimento de apoio social a outros idosos (resposta dicotômica: sim/não).

\section{Procedimento}

A escolha dos participantes foi feita através de uma amostragem não probabilística de residências em bairros onde moram famílias com a renda estabelecida como critério. Pesquisadores foram de porta em porta explicando o objetivo da pesquisa, identificando a existência de algum idoso na família e solicitando a sua participação no estudo. Com os casos que voluntariamente aceitaram participar foram realizadas duas ou três visitas para coletar os dados. Outros lugares onde foram recrutados novos participantes foram feirinhas de artesanato e mercados e vários locais onde havia idosos trabalhando. A escolha dos locais também foi não probabilística e a participação das pessoas foi voluntária e devidamente informada. A coleta de dados foi feita durante duas ou três visitas no local de trabalho de cada participante.

A Escala de Bem-estar Subjetivo e a Escala de Estratégias de Enfrentamento dos Problemas podiam ser respondidas pelo idoso de forma autônoma ou assistida pelo aplicador, enquanto que o instrumento de apoio social e os dados sócio-demográficos foram coletados em forma de entrevista.

\section{Resultados}

Inicialmente foram feitas análises exploratórias dos dados para verificar sua adequação aos pressupostos dos testes estatísticos (Tabachnick \& Fidell, 2001). Os dados faltosos das variáveis contínuas foram substituídos pelas suas respectivas médias. $\mathrm{Na}$ avaliação da normalidade foram identificadas três variáveis com assimetria severa. A transformação inversa de duas delas (idade e pontuação total de satisfação com a vida) melhorou notavelmente as distribuições. A transformação da variável quantidade de apoio social recebido, entretanto, não representou mudança significativa, decidindo-se por retirá-la das análises. Não foram identificados casos extremos multivariados de acordo com a distância Mahalanobis ( $p d "$, 0,001).

Comparações feitas com testes t para amostras emparelhadas entre as três sub-escalas do bem-estar (Tabela 1) mostram que a média da satisfação com a vida $(M=3,92)$ 
foi significativamente maior do que a média dos afetos positivos $(M=3,77 ; t=-10,45 ; p<0,001)$ e dos afetos negativos $(M=3,0 ; t=-12,26 ; p<0,001)$. A mesma com-

Tabela 1

Médias, Desvio Padrão e Resultados das Comparações entre Afetos Positivos, Afetos Negativos, Satisfação com a Vida e Estratégias de Enfrentamento (n=123)

\begin{tabular}{lccc}
\hline Dimensões das Escalas & Média & Desvio Padrão & Teste t \\
\hline$\quad$ Comparação 1 & & & \\
Afetos positivos & 3,27 & 0,67 & $-10,45 * * *$ \\
Satisfação com a vida & 3,92 & 0,59 & $-12,25 * * *$ \\
$\quad$ Comparação 2 & 3,00 & 0,56 & 0,59 \\
Afetos negativos & 3,92 & & $11,16 * * *$ \\
Satisfação com a vida & 3,87 & 0,68 & 0,77 \\
$\quad$ Comparação 3 & 2,90 & &
\end{tabular}

Nota. ${ }^{*} * * *$ p $<0,001$.

Em seguida, análises de regressão hierárquicas foram realizadas para determinar a capacidade preditiva das variáveis apoio social e estratégias de enfrentamento sobre cada uma das três sub-escalas do bem estar subjetivo, uma vez que a influência das variáveis sócio-demográficas tenha sido controlada. Para tanto, o conjunto de variáveis preditoras foi agrupado em três blocos. No primeiro bloco (sócio-demográfico) entraram na equação as variáveis: sexo, contar com pensão, estar trabalhando atualmente, paração entre os resultados dos fatores da escala de enfrentamento revelou que a média do enfrentamento direto e re-avaliativo $(M=3,87)$ é maior do que a média de esquiva $(M=2,90 ; t=-11,65 ; p<0,001)$.

\section{Tabela 2}

Regressão Hierárquica das Variáveis Sócio-Demográficas, de Apoio Social e de Enfrentamento sobre os Índices de Satisfação com a Vida $(n=123)$

\begin{tabular}{|c|c|c|c|c|c|}
\hline Bloco de variáveis & Correlação com VD & $\mathrm{B}$ & $\beta$ & $\mathrm{F}(\mathrm{gl})$ & Valores de $\mathrm{R}$ e $\mathrm{R}^{2}$ \\
\hline \multicolumn{6}{|l|}{ Variáveis sócio-demográficas } \\
\hline Sexo & 0,14 & 0,04 & $0,19^{*}$ & $3,03 *$ & $\mathrm{R}=0,34$ \\
\hline Pensão & 0,23 & 0,05 & $0,23 *$ & $(5,115)$ & $\mathrm{R}^{2}=0,12$ \\
\hline Trabalho & $-0,09$ & 0,01 & 0,06 & & $\mathrm{R}^{2}$ ajus $=0,08$ \\
\hline Escolaridade de $2^{\circ}$ grau ou mais & 0,04 & 0,01 & 0,07 & & $\mathrm{R}^{2}$ mud $=0,12 *$ \\
\hline Idade & 0,21 & 0,16 & 0,19 & & \\
\hline \multicolumn{6}{|l|}{ Variáveis de apoio social } \\
\hline Frequiência de contatos sociais & 0,04 & 0,00 & 0,05 & 7,90 米* & $\mathrm{R}=0,52$ \\
\hline Satisfação com o apoio percebido & 0,23 & 0,00 & 0,25 **** & $(3,112)$ & $\mathrm{R}^{2}=0,27$ \\
\hline Provedor de apoio & 0,38 & 0,07 & 0,34 ***** & & $\begin{array}{l}\mathrm{R}^{2} \text { ajus. }=0,22 \\
\mathrm{R}^{2} \text { mud. }=0,15^{* * *} *\end{array}$ \\
\hline \multicolumn{6}{|l|}{ Variáveis de enfrentamento } \\
\hline Enfrentamento direto e re-avaliativo & 0,43 & 0,05 & 0,37 ***** & 9,78 **** & $\mathrm{R}=0,62$ \\
\hline Enfrentamento de esquiva & $-0,14$ & $-0,01$ & $-0,09$ & $(2,110)$ & $\begin{array}{l}\mathrm{R}^{2}=0,38 \\
\mathrm{R}^{2} \text { ajus. }=0,32 \\
\mathrm{R}^{2} \text { mud. }=0,11^{*} * * *\end{array}$ \\
\hline
\end{tabular}

Notas. ${ }^{*} \mathrm{p}<0,05 \quad$ *** $\mathrm{p}<0,01 \quad$ **** $\mathrm{p}<0,001$.

escolaridade (segundo grau completo ou mais) e idade. Excetuando a idade, todas as demais variáveis foram codificadas como variáveis dummy ${ }^{1}$. No segundo bloco (apoio social) foram incluídas as variáveis: freqüência de contatos sociais, satisfação com o apoio recebido e apoio que o idoso provê aos demais (variável dummy). No terceiro e último bloco (estratégias de enfrentamento) foram incluídas as variáveis: enfrentamento direto e re-avaliativo e enfrentamento de esquiva.

\footnotetext{
${ }^{1}$ Código binário utilizado para representar uma variável
discreta em análises de regressão. Na codificação dummy cada
variável discreta é representada por K-1 variáveis dicotomas,
onde K é igual ao número de níveis da variável discreta. Dessa
forma, a variável sexo, por exemplo, é representada por uma

${ }^{1}$ Código binário utilizado para representar uma variável
discreta em análises de regressão. Na codificação dummy cada
variável discreta é representada por K-1 variáveis dicotomas,
onde K é igual ao número de níveis da variável discreta. Dessa
forma, a variável sexo, por exemplo, é representada por uma

${ }^{1}$ Código binário utilizado para representar uma variável
discreta em análises de regressão. Na codificação dummy cada
variável discreta é representada por K-1 variáveis dicotomas,
onde K é igual ao número de níveis da variável discreta. Dessa
forma, a variável sexo, por exemplo, é representada por uma

${ }^{1}$ Código binário utilizado para representar uma variável
discreta em análises de regressão. Na codificação dummy cada
variável discreta é representada por K-1 variáveis dicotomas,
onde K é igual ao número de níveis da variável discreta. Dessa
forma, a variável sexo, por exemplo, é representada por uma

${ }^{1}$ Código binário utilizado para representar uma variável
discreta em análises de regressão. Na codificação dummy cada
variável discreta é representada por K-1 variáveis dicotomas,
onde K é igual ao número de níveis da variável discreta. Dessa
forma, a variável sexo, por exemplo, é representada por uma
}

única variável $(2-1=1)$ onde masculino pode ser codificado como zero e feminino como 1 . Cada uma das $\mathrm{K}-1$ variáveis dummyrepresenta um contraste entre um dos níveis da variável discreta e o nível de referência escolhido pelo pesquisador. Ver mais detalhes em Tabachnick e Fidell (2001). 
A Tabela 2 mostra os resultados da regressão hierárquica das variáveis independentes sobre a sub-escala satisfação com a vida. No primeiro bloco, as variáveis sócio-demográficas apresentaram uma capacidade preditiva significativa sobre a satisfação com a vida $\left[R^{2}=0,12\right.$; $F(5,115)=3,03, p<0,01]$. Em seguida, o segundo bloco, com as variáveis relativas ao apoio social, também apresentou uma predição significativa $\left[R^{2}=0,27 ; F(3,112)=\right.$ $7,90, p<0,001]$. Por último, e uma vez controladas as variáveis dos dois blocos anteriores, a adição das estratégias de enfrentamento na equação resultou num incremento significativo na percentagem da variância da satisfação com a vida $\left[R^{2}=0,38 ; F(2,110)=6,77, p<0,001\right]$. O modelo final ficou com cinco preditores positivos: sexo, pensão, satisfação com o apoio recebido, apoio oferecido e enfrentamento direto e re-avaliativo. Isto é, ser mulher, ter pensão, maior satisfação com o apoio, ser provedor de apoio e maior uso da estratégia de enfrentamento direto e re-avaliativo, prediz maiores pontuações da satisfação com a vida.
A Tabela 3 mostra os resultados do mesmo tipo de regressão hierárquica com as variáveis relativas à sub-escala de afetos positivos. Ao contrário da análise com a subescala satisfação com a vida, o bloco das variáveis sóciodemográficas não apresentou predição significativa sobre os afetos positivos. Já no segundo bloco, com o conjunto de variáveis do apoio social, a quantidade de variância predita foi significativa $\left[R^{2}=0,14 ; F(3,112)=3,08, p<0,05\right]$. Finalmente, também foi observado um incremento significativo na quantidade de variância predita após a entrada das variáveis de enfrentamento no terceiro bloco $\left[R^{2}=\right.$ o,34, $F(2,110)=15,20, p<0,001]$. O modelo final ficou com $34 \%$ da variância explicada dos afetos positivos, tendo como preditores positivos à satisfação com o apoio e o enfrentamento direto e re-avaliativo, enquanto que o enfrentamento esquivo foi preditor negativo. Quando aumenta a satisfação com o apoio recebido e o uso da estratégia de enfrentamento direto e re-avaliativo, aumentam também os afetos positivos. Inversamente, quando aumenta o uso da estratégia de enfrentamento de esquiva, diminuem os afetos positivos.

Tabela 3

Regressão Hierárquica das Variáveis Sócio-Demográficas, de Apoio Social e de Enfrentamento sobre os Índices de Afetos Positivos $(n=123)$

\begin{tabular}{|c|c|c|c|c|c|}
\hline Bloco de variáveis & Correlação com VD & B & $\mathrm{b}$ & $\mathrm{F}(\mathrm{gl})$ & Valores de $\mathrm{R}$ e $\mathrm{R}^{2}$ \\
\hline \multicolumn{6}{|l|}{ Variáveis sócio-demográficas } \\
\hline Sexo & 0,17 & 0,26 & 0,20 & 1,74 & $\mathrm{R}=0,26$ \\
\hline Pensão & $-0,05$ & 0,00 & 0,02 & $(5,115)$ & $\mathrm{R}^{2}=0,07$ \\
\hline Trabalho & 0,11 & 0,25 & 0,19 & & $\mathrm{R}^{2}$ ajus. $=0,03$ \\
\hline Escolaridade de $2^{\circ}$ grau ou mais & 0,07 & 0,14 & 0,11 & & $\mathrm{R}^{2}$ mud $=0,07$ \\
\hline Idade & 0,05 & 80,41 & 0,15 & & \\
\hline
\end{tabular}

Variáveis de apoio social

\begin{tabular}{|c|c|c|c|c|c|}
\hline $\begin{array}{l}\text { Frequiência de contatos sociais } \\
\text { Satisfação com o apoio percebido } \\
\text { Provedor de apoio }\end{array}$ & $\begin{array}{l}0,03 \\
0,20 \\
0,16\end{array}$ & $\begin{array}{l}0,00 \\
0,02 \\
0,20\end{array}$ & $\begin{array}{l}-0,12 \\
0,25^{* * * *} \\
0,15\end{array}$ & $\begin{array}{l}3,08 * \\
(3,112)\end{array}$ & $\begin{array}{l}\mathrm{R}=0,38 \\
\mathrm{R}^{2}=0,14 \\
\mathrm{R}^{2} \text { ajus. }=0,08 \\
\mathrm{R}^{2} \text { mud. }=0,07 *\end{array}$ \\
\hline \multicolumn{6}{|l|}{ Variáveis de enfrentamento } \\
\hline $\begin{array}{l}\text { Enfrentamento direto e re-avaliativo } \\
\text { Enfrentamento de esquiva }\end{array}$ & $\begin{array}{l}0,38 \\
-0,24\end{array}$ & $\begin{array}{l}0,41 \\
-0,26\end{array}$ & $\begin{array}{l}0,43 \text { ***** } \\
-0,31 * * * * 1\end{array}$ & $\begin{array}{l}15,20 * * * * \\
(2,110)\end{array}$ & $\begin{array}{l}\mathrm{R}=0,58 \\
\mathrm{R}^{2}=0,34 \\
\mathrm{R}^{2} \text { ajus. }=0,28 \\
\mathrm{R}^{2} \text { mud. }=0,19 * * *\end{array}$ \\
\hline
\end{tabular}

Notas. ${ }^{*} \mathrm{p}<0,05 \quad{ }^{*} \mathrm{*} \mathrm{p}<0,01 \quad$ ***** $\mathrm{p}<0,001$

As variáveis sócio-demográficas também não contribuíram significativamente para a explicação das diferenças dos afetos negativos (ver Tabela 4). A entrada do segundo bloco, apoio social, explicou 12\% variância dos afetos negativos $[F(3,112)=3,32, p<0,01]$. Por fim, o resultado final, com todos os blocos na equação de regressão, mostrou uma quantidade significativa de variância sendo predita no fator afetos negativos $\left[R^{2}=0,17 ; F(2,110)=2,98\right.$, $p<0,05]$. Este modelo teve como preditor negativo o apoio provido e como preditor positivo o enfrentamento de esquiva. As pessoas que são provedoras de apoio apresentaram menores pontuações de afetos negativos, enquanto que as pessoas com maiores pontuações de enfrentamento de esquiva, relataram mais afetos negativos. 
Psicologia: Reflexão e Crítica, 19(2), 301-308.

Tabela 4

Regressão Hierárquica das Variáveis Sócio-Demográficas, de Apoio Social e de Enfrentamento sobre os Índices de Afetos Negativos $(n=123)$

\begin{tabular}{|c|c|c|c|c|c|}
\hline Bloco de variáveis & Correlação com VD & $\mathrm{B}$ & $\mathrm{b}$ & $\mathrm{F}(\mathrm{gl})$ & pres de $\mathrm{R}$ e $\mathrm{R}^{2}$ \\
\hline \multicolumn{6}{|l|}{ Variáveis sócio-demográficas } \\
\hline Sexo & 0,06 & 0,05 & 0,05 & \multirow{5}{*}{$\begin{array}{l}1,04 \\
(5,115)\end{array}$} & $\mathrm{R}=0,21$ \\
\hline Pensão & $-0,14$ & $-0,14$ & $-0,12$ & & $\mathrm{R}^{2}=0,04$ \\
\hline Trabalho & 0,04 & $-0,04$ & $-0,04$ & & $\mathrm{R}^{2}$ ajus. $=0,00$ \\
\hline Escolaridade de $2^{\circ}$ grau ou mais & 0,09 & 0,10 & 0,09 & & $\mathrm{R}^{2}$ mud. $=0,04$ \\
\hline Idade & $-0,14$ & $-53,21$ & $-0,12$ & & \\
\hline \multicolumn{6}{|l|}{ Variáveis de apoio social } \\
\hline Frequiência de contatos sociais & 0,06 & 0,00 & 0,03 & \multirow{4}{*}{$\begin{array}{l}3,32 \text { ** } \\
(3,112)\end{array}$} & $\mathrm{R}=0,35$ \\
\hline Satisfação com o apoio percebido & 0,16 & 0,01 & 0,14 & & $\mathrm{R}^{2}=0,12$ \\
\hline Provedor de apoio & $-0,26$ & $-0,28$ & $-0,29 *$ & & $\mathrm{R}^{2}$ ajus. $=0,06$ \\
\hline & & & & & $\mathrm{R}^{2}$ mud. $=0,08 * *$ \\
\hline \multicolumn{6}{|l|}{ Variáveis de enfrentamento } \\
\hline Enfrentamento direto e re-avaliativo & $-0,07$ & $-0,05$ & $-0,06$ & $2,98^{*}$ & $\mathrm{R}=0,41$ \\
\hline \multirow{3}{*}{ Enfrentamento de esquiva } & \multirow[t]{3}{*}{0,24} & \multirow[t]{3}{*}{0,17} & $0,24 *$ & \multirow{3}{*}{$(2,110)$} & $\mathrm{R}^{2}=0,17$ \\
\hline & & & & & $\mathrm{R}^{2}$ ajus. $=0,09$ \\
\hline & & & & & $\mathrm{R}^{2} \mathrm{mud}=0,04 *$ \\
\hline
\end{tabular}

Notas. ${ }^{*} \mathrm{p}<0,05 \quad$ * $*$ $\mathrm{p}<0,01 \quad$ **** $\mathrm{p}<0,001$.

\section{Discussão}

As diferenças significativas entre as sub-escalas do bemestar subjetivo corroboram a importância da distinção dos componentes afetivo e cognitivo na interpretação do bemestar subjetivo também na velhice. Pesquisas indicam que a satisfação com a vida (componente cognitivo) aumenta com a idade enquanto que a intensidade das experiências afetivas diminui no decorrer dos anos (Diener, Suh, Lucas \& Smith, 1999; Kunzmann, Little \& Smith, 2000). Na amostra estudada, a média das pontuações da satisfação com a vida foi significativamente maior do que a média dos afetos positivos e dos afetos negativos.

Várias explicações têm sido sugeridas sobre a relação diferencial entre a idade e o bem-estar subjetivo. Herzog, Rodgers e Woodworth (1981), por exemplo, propõem explicações relativas a processos subjetivos de ajuste, tais como a diminuição da discrepância entre o que é esperado e o que é obtido na vida. Chatters (1988) também sugere que aqueles idosos que não têm grandes perdas funcionais se julgam em uma situação satisfatória ao se compararem com quem tem perdas maiores. Diener et al. (1999) propõem que, na velhice, diminui a intensidade emocional com a qual são sentidas as experiências da vida. Para Pinqüart e Sörensen (2000) a satisfação reflete uma relativa estabilidade nos julgamentos das experiências obtidas ao longo da vida. Já a vivência afetiva reflete avaliações em curto prazo, conseqüências de variabilidades situacionais.

A amostra de idosos analisada neste estudo não apresentava grandes perdas de capacidade funcional. Embora a ausência de uma avaliação específica - através de um instrumento de diagnóstico geriátrico válido -, possa ser considerada como uma limitação do presente estudo, as observações feitas in loco permitem afirmar que as condições de saúde dos entrevistados eram relativamente positivas (foram observados deslocamentos físicos autônomos, boas condições cognitivas, auditivas e de fala que permitiram a realização das várias entrevistas). Tais idosos, por apresentarem boas condições de saúde, tendem a avaliar mais positivamente seu bem estar por conta do contraste evidente da sua condição com a dos que estão em piores condições (Chatters, 1988).

Quanto às estratégias de enfrentamento, os idosos recorreram mais à estratégia direta e re-avaliativa do que à estratégia de esquiva, comprovando que os idosos utilizam mais as re-avaliações positivas para enfrentar condições estressantes do que as pessoas mais jovens (Diehl, Coyle \& Labouvie-Vief, 1996). Wrosch, Heckhausen e Lachman (2000) também sugerem que as avaliações positivas dos problemas são características da velhice porque funcionam como um mecanismo compensatório frente às possíveis perdas que o idoso possa estar experimentando nesta etapa da vida. Finalmente, Hanson e Mintz (1997) encontraram altos níveis de enfrentamento relacionados à solução do problema e à saúde psicológica e conservação da autonomia.

Segundo Pratt e Norris (1994) os idosos utilizam menos a evasão do que os jovens. Os autores sugerem que os idosos recorrem principalmente a dois tipos de estratégias para manejar as mudanças relacionadas com a idade: (a) comportamentos instrumentais focalizados na prevenção e alívio das perdas que podem influenciar negativamente a própria identidade e, (b) processos de acomodação que 
procuram alterar fins e concepções através da revalorização - para fazê-las mais consistentes com as perdas no funcionamento pessoal. Dessa forma, os idosos utilizam métodos de acomodação como uma estratégia que reflete a flexibilidade e adaptação, mas não resignação ou esquiva.

Todos estes resultados podem ajudar na interpretação da relação preditiva entre as estratégias de enfrentamento e o bem-estar subjetivo verificada na amostra estudada. A estratégia de enfrentamento direto e re-avaliativo prediz positivamente a satisfação com a vida e os afetos positivos e o enfrentamento de esquiva prediz negativamente os afetos positivos e positivamente aos afetos negativos. Resultados nessa direção também foram encontrados por Aldwin et al. (1996) indicando que as pessoas idosas, que utilizam ações instrumentais para resolver seus problemas, percebem mais resultados positivos. Similarmente, Pruchno, Burant e Peters (1997) encontraram que as pessoas que recorrem às estratégias instrumentais, freqüentemente percebem mais afetos positivos e menos sintomas de distúrbios psicológicos. Hanson e Mintz (1997) constataram que os idosos que enfrentam seus problemas com orientação à solução relatam maior bem-estar, afetos positivos, autonomia, relações positivas com as pessoas e menos depressão e ansiedade.

Outras evidências também apóiam os resultados deste estudo. Aldwin et al. (1996) encontraram que os idosos que recorrem ao estilo de evasão têm maior probabilidade de ter resultados e sentimentos negativos, em comparação com as pessoas que utilizam a solução direta dos problemas. Numa amostra de idosos viúvos, Voyer e Vézina (1995) identificaram que a ameaça à auto-estima e o enfrentamento de esquiva explicaram até $65 \%$ da variância da tensão psicológica. Pode-se concluir, portanto, que as estratégias de esquiva aos problemas (tais como a evasão, negação da realidade e a auto-distração) representam mais uma desvantagem do que um ganho para as pessoas.

Também se confirmou o poder preditivo do apoio social no bem-estar dos idosos: (a) a satisfação com a vida aumenta com a satisfação com o apoio percebido e com o apoio provido; (b) os afetos positivos aumentam com a satisfação com o apoio e; (c) os afetos negativos diminuem quando o idoso provê apoio aos outros. Este poder preditivo, entretanto, não pode ser generalizado para todas as dimensões do apoio social. Uma alta freqüência de contatos com a rede social, por exemplo, não contribuiu significativamente na predição do bem-estar. Em concordância com o indicado por outros pesquisadores (Abril, 1998; Gracia, 1997), apenas os aspectos funcionais do apoio social figuraram como preditores significativos. Uma maior freqüência de contatos, através das redes sociais, aumenta a probabilidade de que aconteçam intercâmbios sociais, mas, efeitos propriamente protetores à saúde, são devidos à natureza desses intercâmbios, ou seja, às funções do apoio, bem como aos aspectos subjetivos envolvidos.

Não somente as qualidades do apoio social recebido estão relacionadas ao bem-estar subjetivo: dar apoio aos demais também é importante (Jung, 1997). Segundo dados do IBGE (2002), 62,4\% dos idosos no Brasil é consi- derado pelos próprios membros da família, como responsáveis pelo domicilio em que vivem. Essa situação mostra a importância do papel do idoso na manutenção da família e cria um contexto para entender as dinâmicas de apoio recíproco entre o idoso e sua família. Os dados desta pesquisa indicaram que ser provedor de apoio contribuiu significativamente para o aumento da satisfação do idoso com a vida e para a diminuição dos seus afetos negativos.

A reciprocidade do apoio recebido e provido revela um nível satisfatório de autonomia e esta capacidade funcional pode ser um moderador do impacto da estratégia direta e re-avaliativa e do apoio dado sobre o bem-estar dos idosos. Tal constatação permite olhar a velhice com uma perspectiva diferente, pois, em geral, presume-se que o idoso é quem precisa do apoio para satisfazer suas necessidades (Gracia, 1997). Mas os resultados indicam que os idosos que enfrentam em forma direta e re-avaliativa seus problemas, recebem e dão apoio, são os que relatam maior bem-estar. Isso contribui para mudar a concepção do idoso como mero receptor passivo de ajuda, indicando a possibilidade de seu envolvimento bidirecional, dando e recebendo recursos para a promoção do próprio bem-estar.

A relação preditiva significativa das variáveis sóciodemográficas sobre a satisfação com a vida, mas não sobre os afetos positivos e negativos, comprovam o baixo poder explicativo destes indicadores no bem-estar subjetivo (Andrews \& Robinson, 1991; Bohlander, 1999). No entanto, características da amostra estudada sugerem cautela quanto à generalização do que foi observado. A amostra analisada foi composta por pessoas alfabetizadas funcionais com 60 anos ou mais, moradores de uma cidade litorânea de nível médio para grande, com uma renda familiar que permite satisfazer suas necessidades básicas de saúde, alimentação, moradia e vestuário.

Os resultados mostram a importância de se analisar separadamente às dimensões cognitiva e afetiva do bem-estar subjetivo na terceira idade. Estas dimensões não apenas se manifestam com intensidades diferenciadas, como possuem relações também distintas com o apoio social e as estratégias de enfrentamento. Dos três componentes do bem-estar subjetivo, satisfação com a vida foi o que mostrou a maior relação preditiva com o apoio social e com as estratégias de enfrentamento. A satisfação com a vida é maior nas mulheres, nas pessoas que recebem pensão, nas pessoas que estão satisfeitas com o apoio recebido, nas pessoas que dão apoio aos outros e nas pessoas que enfrentam os problemas de forma direta e através de uma re-avaliação positiva. Já os afetos positivos também aumentam com a satisfação do apoio recebido e com o enfrentamento direto e re-avaliativo, bem como com a diminuição do enfrentamento de esquiva.

Os afetos negativos diminuem quando se fornece apoio social embora aumentem com o enfrentamento de esquiva. Mais uma razão para que não se considere o idoso como apenas um receptor passivo de ajuda e compreensão. $\mathrm{O}$ alcance de suas ações também contribui para seu bem estar e satisfação. Afinal, o menor poder preditivo do apoio social e dos estilos de enfrentamento, sobre seus afetos 
positivos e negativos, indica que outras variáveis estão envolvidas nas dimensões do bem-estar subjetivo do idoso, particularmente o que estiver diretamente ligado a sua autonomia funcional.

\section{Referências}

Abril, V. (1998). Instrumentos de psicología comunitaria. Apoyo social y marketing social. Valencia, España: Promolibro.

Albuquerque, A. S., \& Tróccoli, B. T. (2004). Desenvolvimento de uma escala de bem-estar subjetivo. Psicologia: Teoria e Pesquisa, 20, 153-164.

Aldwin, C., Sutton, K., \& Lachman, M. (1996). The development of coping resources in adulthood. Journal of Personality, 64, 837-871.

Andrews, F., \& Robinson, J. (1991). Measures of subjetive wellbeing. In J. P. Robinson, P. R. Shaver \& L. S. Wrihtsman (Eds.), Measures of personality and social psychological attitudes (Vol. 1, pp. 61-114). San Diego, CA: Academic Press.

Anguas, A. (1997). El significado del bienestar subjetivo, su valoración en México. Disertación de Maestría, Universidad Nacional Autónoma de México.

Agulló, M. (1999). Mayores, actividad y trabajo en el proceso de envejecimiento y jubilación: Una aproximación psico-sociológica. Disertación de Doctorado, Universidad Complutense de Madrid, España.

Bohlander, R. (1999). Differentiation of self, need fulfillment and psychological well-being in married men. Psychological Reports, 84, 1274-1280.

Chatters, L. (1988). Subjective well-being evaluations among older black Americans. Psychology and Aging, 13, 184-190.

Diehl, M., Coyle, N., \& Labouvie-Vief, G. (1996). Age and sex differences in strategies of coping and defense across the life span. Psychology and Aging, 11, 127-139.

Diener, E., Suh, E., Lucas, R., \& Smith, H. (1999). Subjective well-being: Three decades of progress. Psychological Bulletin, 125, 276-302.

Emmons, R. (1986). Personal strivings: An approach to personality and subjective well-being. Journal of Personality and Social Psychology, 51, 1058-1068.

Felton, B., \& Shinn, M. (1992). Social integration and social support: Moving "social support" beyond the individual level. Journal of Community Psychology, 20, 103-115.

García-Sanz, B., \& Saco, A. (1995). Envejecimiento de la población mundial. Temas para el Debate, 11, 35-38.

Gracia, E. (1997). El apoyo social en la intervención comunitaria. Barcelona, España: Paidós.

Hanson, K. M., \& Mintz, L. B. (1997). Psychological health and problem-solving self-appraisal in older adults. Journal of Counseling Psychology, 44, 433-441.

Herzog, A., Rodgers, W., \& Woodworth, J. (1981). Subjective wellbeing among different age groups. Ann Arbor, MI: University of Michigan.

Instituto Brasileiro de Geografia e Estatística. (2002). Perfil dos idosos responsáveis pelos domicílios no Brasil. 2000. Estudos e Pesquisas. Informação Demográfica e Socioeconômica (Vol. 9). Rio de Janeiro, RJ: Autor.

Jung, J. (1997). Balance and source of social support in relation well-being. The Journal of General Psychology, 724, 77-90.

Krause, N., \& Borawski-Clark, E. (1995). Social class differences in social support among older adults. The Gerontologist, 35, 498-508.
Krause, N., Ingersoll-Dayton, B., Ellison, C., \& Wulf, K. (1999). Aging, religious doubt and psychological well-being. The Gerontologist, 39, 525-533.

Kunzmann, U., Little, T., \& Smith, J. (2000). Is age-related stability of subjective well-being a paradox? Cross-sectional and longitudinal evidence from Berlin Aging Study. Psychology and Aging; 15, 511-526.

Martínez, M., \& García, M. (1994). La autopercepción de la salud y el bienestar psicológico como indicador de calidad de vida percibida en la vejez. Revista de Psicología de la Salud, $6,55-74$.

Ministério da Saúde. (2001). Retirado em maio de 2001, em http/ /www.saude.gov.br/programas/fisica/ fisica.htm

Newson, J., \& Schultz, R. (1996). Social support as a mediator in the relation between functional status and quality of life in older adults. Psychology and Aging, 11, 34-44.

Ormel, J., Linderber, S., Steverink, N., \& Vonkorf, M. (1997). Quality of life and social production functions: A framework for understanding health effects. Social Science Medical, 4.5, 1051-1063.

Pinquiart, M., \& Sörensen, S. (2000). Influence of socioeconomic status, social network, and competence on subjective wellbeig in later life: A meta-analysis. Psychology and Aging, 15(2), 187-224.

Pratt, M., \& Norris, J. (1994). The social psychology of aging: Waltham, MA: Blackwell.

Pruchno, R., Burant, C., \& Peters, N. (1997). Coping strategies of people living in multigenerational households: Effects on well-being. Psychology and Aging, 12, 115-124.

Ranzjin, R., \& Luszcz, M. (2000). Measurement of subjective quality of life of elders. International Journal of Aging and Human Development, 50, 263-278.

Tabachnick, B., \& Fidell, L. (2001). Using multivariate statistics. Fourth Edition. New York: Harper Collins.

Voyer, M., \& Vézina, J. (1995). Contribution of hassles, appraisal and coping to psychological distress among elderly widows. Canadian Journal on Aging, 4, 498-5 10.

Wrosch, C., Heckhausen, J., \& Lachman, M. (2000). Primary and secondary control strategies for managing health and financial stress across adulthood. Psychology and Aging; 15, 387-399.
Recebido: 01/09/2004 1 revisão: 01/03/2005 Aceite final: 25/05/2005 